\title{
Inferencias sobre un texto narrativo en contextos de interacción en la educación inicial*
}

\section{Inferences over a narrative text in contexts of interaction in early education}

Recibido: julio 26 de 2010 | Revisado: enero 24 de 2011 |Aceptado: marzo 16 de 2011

\author{
Claudia Patricia DuQue Aristizábal ** \\ Universidad del Tolima, Colombia \\ Miralba Correa Restrepo **** \\ Universidad del Valle, Cali, Colombia
}

\section{RESUMEN}

La investigación que da lugar al presente artículo se enmarca en la Psicología Educativa de corte cultural. Explora las relaciones entre las características de las interacciones establecidas para propiciar la interpretación de un texto narrativo y las inferencias que los niños hacen sobre él. Un total de 44 niños repartidos en cuatro grupos de preescolar participó en el estudio. El diseño es de tipo descriptivo-exploratorio, con dos clases de análisis: cualitativo -análisis del discurso- y cuantitativo -análisis de redes sociales-, para procesar los datos. Se encontró que los niños cuyas maestras propician más interacciones cognitivas de alta demanda, proponiendo el análisis de los aspectos implícitos en el texto, logran mayor elaboración inferencial, mientras que los niños cuyas docentes proponen menos interacciones con discusiones principalmente sobre información explícita en el texto, hacen pocas inferencias y con nivel de menor elaboración.

Palabras clave autores

Interacción, comprensión textual, inferencias, educación inicial, textos narrativos. Palabras clave descriptores

Psicología educativa, invetigación mixta, diseño descriptivo-exploratorio, niños. contextos de interacción en la educación inicial Universitas Psychologica, 11(2), 559-570.

* El artículo se origina en la investigación "Inferencias sobre un texto narrativo en contextos de interacción en la educación inicial". Tesis de Maestría en Psicología, Universidad del Valle, Grupo Lenguaje, Cognición y Educación. Directora de tesis: Miralba Correa Restrepo.

** Facultad de Ciencias de la Educación, Departamento de Psicopedagogía. Universidad del Tolima, Barrio Santa Helena Parte Alta, Ibagué-Tolima E-mail:, claduque@yahoo.com. ResearcherID: Duque, C. F-3468-2012.

**** Instituto de Psicología, Universidad del Valle. Edificio 388. Cuarto Piso. Ciudadela Universitaria Meléndez. Grupo de Investigación Lenguaje, CogniciónyEducación.E-mail: miralbacorrea@yahoo. com. ResearcherID: Correa, M. F-4024-2012

\begin{abstract}
A B S T R A C T
This research explores the relationship between the characteristics of interactions established to favor the narrative text interpretation and the inferences that children make on it. This is marked in the educative, cognitive and cultural psychologist. In this research participated four groups of kinder garden with a total of forty four children. This was a descriptive and explorative design. Was realized a discursive analysis and a social nets analysis to process information. It was find that children which teachers favor more interactions and better interactions raised a textual analyses got a high inferencial elaboration, and children which teachers proposed low interaction raised discussions about explicit information in the text, realized a few inferences and with a low level of elaboration.

Key words authors

Interaction, textual comprehension, inferences, initial education, narrative text. Key words plus

Educative psychology, mixed research, descriptive-exploratory design, children.
\end{abstract}




\section{Introducción}

Los cambios que se han dado en las concepciones sobre el funcionamiento cognitivo en la primera infancia, parten de afirmar que los niños piensan y piensan bien; además, son capaces de interrogarse sobre los fenómenos del mundo y sobre diferentes manifestaciones culturales, entre ellas el lenguaje escrito (Correa, 2003; Orozco, 2003). Estos cambios han repercutido en la manera de estudiar la comprensión de los textos, poniendo de relieve el interés por los funcionamientos cognitivos involucrados.

Así como se destacan las enormes competencias de los niños pequeños, también se postula la incidencia de las interacciones en la posibilidad de mejorar la interpretación de los textos. La comprensión de textos narrativos, cuya condición es la inferencia, requiere que se den interacciones, como las que ocurren en la lectura compartida en la que la maestra realiza preguntas que permiten a los niños aproximarse a las situaciones y a los personajes propuestos en el texto, vinculándolos con la enciclopedia que ellos hayan constituido a partir de sus experiencias cotidianas, ligadas con la vida diaria y con la lectura.

Las primeras experiencias de los niños con la lectura son fundamentales en la forma como ellos la asumen posteriormente (Bobbitt, 2001; Bravo, Villalón \& Orellana, 2004, 2006; Duque, 2006; Duque, Vera \& Hernández, 2010; Saracho, 2002; Stahl \& Yaden, 2004; Teberosky \& Tolchinsky, 1998).

Entre las prácticas universalmente reconocidas en la enseñanza de la lectura en educación inicial, se encuentran las de leer cuentos, escritos por literatos reconocidos (Correa, 2003). De acuerdo con Van Lizendoorn y Pellegrini (1995 citados por Flórez, Torrado, Areválo, Quintero \& Rodríguez, 2008) y Bruner $(1995,1997)$, este tipo de textos favorece el desarrollo cognitivo del niño por varias razones: recrean experiencias vividas, concuerdan con los funcionamientos metafóricos del pensamiento infantil y generan el asombro de los niños, tanto por las situaciones que plantean como por el uso de formas de discurso que los obligan a hacerse preguntas para comprender lo contado.
Comparativamente con otros textos, los narrativos posibilitan la elaboración de una gran variedad de inferencias, ya que demandan una comprensión que requiere generar un "modelo de situación". A este respecto, Van Dijk y Kintsch (1983) señalan: "Un modelo de situación se define como la representación cognitiva de los acontecimientos, acciones, personas y, en general, de la situación sobre la que trata el texto" (p. 12). Así mismo, las inferencias son definidas como representaciones mentales que el lector/oyente construye o añade al comprender el texto, a partir de las aplicaciones de sus propios conocimientos a las indicaciones explícitas en el mensaje (Belinchón, Rivière \& Igoa 2000; Graesser \& Wiemer-Hastings, 1999; Jurado, Bustamante \& Pérez, 1998).

La comprensión inferencial no solo permite leer lo implícito en el texto para lograr un esclarecimiento de su estructura profunda, sino que propicia el desarrollo de competencias para desenvolverse en la vida y en diversas áreas de conocimiento (Scardamalia \& Bereiter, 1992). Asumir la comprensión textual como un proceso que implica inferir, lleva a una concepción de lectura interactiva y transaccional (Cerrillo, Larrañaga \& Yubero, 2002; Eco, 1996; Greimas \& Courtés, 1993 citados por Correa, 2003).

Desde la perspectiva cognitiva constructivista, se asume que cuando se lee un texto narrativo se pueden generar varios tipos de inferencias. De este modo, las inferencias que se realizan durante el proceso de comprensión incluirían, por un lado, las que establecen la coherencia local, denominadas referenciales y de antecedentes causales y, por otro, las que aseguran la coherencia global, como las inferencias que proporcionan la meta superordinada, las inferencias temáticas, o aquellas que ayudan a configurar la reacción emocional del personaje. Por otra parte, entre las inferencias que se generan con posterioridad a la lectura, se encuentran las consecuentes causales, las pragmáticas, las instrumentales y las predictivas (Graesser \& Zwaan, 1995; Jouini, 2005; León, 2001; Orozco, 2003; Orozco, Puche, Millán \& Rojas, 2004).

La posibilidad de elaborar inferencias, es algo que los niños pueden hacer desde muy temprana 
edad. Puche (2003 citada por Orozco et al., 2004) encuentra que desde los cuatro años, el funcionamiento inferencial parece formar parte de la actividad mental del niño al enfrentarse a los textos. Igualmente, Duque y Vera (2010) dan cuenta en un estudio exploratorio de que los niños de cinco años de preescolar están en capacidad de formular inferencias de menor complejidad. Otros estudios muestran que los niños menores de 6 años tienen una gran competencia para elaborar inferencias emocionales y causales (Goldman, Varma, Sharp $\&$ Cognition and Technology Group at Vanderbilt, 1999; Jiménez \& Marmolejo, 2007; Ordóñez \& Bustamante, 2000; Perrusi \& Galvao, 1998; Espéret \& Fayol, 1997).

Si bien se puede decir que la inferencia es un funcionamiento cognitivo del sujeto y como tal es propia de cada individuo, también es posible afirmar que en el espacio escolar las interacciones pueden incrementar las inferencias que los niños realizan. Esta presunción es posible si se considera que dentro de los aspectos que determinan la comprensión inferencial que los niños logran de un texto, se encuentra la interacción con otros niños y con la maestra, y que el tipo de discurso que esta utiliza, la calidad de sus observaciones sobre los diferentes aspectos del cuento y el tipo de preguntas que dirige a los niños juegan un papel central en la elaboración de inferencias.

Desde la práctica textual ${ }^{1}$ y la Psicología Cultural, se concibe la interacción como una relación dinámica en la que se inscribe el hecho de enseñar y aprender, procesos que implican dos sujetos con diferentes niveles de conocimiento, donde cualquiera de los participantes trata de hacer saber algo al otro y de hacer que el otro haga algo (Correa, Orozco \& Conde, 1999). Durante la interacción se da el intercambio de papeles que ocurre en la medida en que se establece una acción conjunta entre el maestro y el sujeto que aprende, en relación con un

1 Esta es una propuesta adelantada por Miralba Correa Restrepo, que se apoya en principios procedentes de la semiótica y la psicolingüística; desde esta propuesta, se aborda la lectura y la escritura con una concepción del lenguaje como significación y de su aprendizaje como la construcción de sentidos por parte del sujeto que aprende. objeto de conocimiento particular (Bruner, 1997; Correa, 2006; Mercer, 2001), que bien puede ser la comprensión o la producción textual.

Estudios como el de González (2005) y Moschovaki y Meadows (2005) presentan resultados empíricos que apoyan la presunción de que, en el espacio escolar, ciertas características de las interacciones pueden incrementar el funcionamiento cognitivo de los niños. En ambas investigaciones hay un interés por las interacciones relacionadas con el funcionamiento cognitivo de los participantes alrededor del texto narrativo, y tienen en cuenta la comprensión textual y las interacciones de manera conjunta, en niños escolarizados entre 3 y 6 años.

Tanto las inferencias como las interacciones han sido abordadas de manera separada dentro de marcos explicativos en la disciplina psicológica (Espéret \& Fayol, 1997; Perrusi \& Galvao, 1998; Rincón, Narváez \& Roldán, 2005); algunos teóricos, sin embargo, se han interesado por los dos aspectos de manera articulada (González, 2005; Moschovaki \& Meadows, 2005). Específicamente en Colombia, hay ausencia de estudios publicados que reúnan estas dos dimensiones, por lo que integrarlos en una investigación permite avanzar en la comprensión del funcionamiento cognitivo de los niños y en las prácticas pedagógicas en el aula, que facilitan la comprensión textual. Es con base en las afirmaciones anteriores que se formula la siguiente pregunta: iqué relación existe entre las inferencias hechas por los niños de preescolar sobre un texto narrativo y las características de las interacciones docente-niños y niños-niños que ocurren en el aula de clase en conexión con ese texto?

Para sustentar conceptualmente la investigación, se revisaron las propuestas contemporáneas que sobre interacción, inferencias, lectura y comprensión de textos narrativos se han presentado desde diferentes aproximaciones (Bruner, 1995, 1997; Eco, 1996; Goodman, 1991; Graesser \& Wiemer-Hastings, 1999; Graesser \& Zwaan, 1995; Mercer, 2001; Zwaan \& Madden, 2004; Zwaan, van den Broek \& Sundermeier, 2005; Teberosky $\&$ Tolchinsky, 1998). El estudio se inscribe en la psicología del lenguaje, cultural y educativa, y releva 
el conocimiento compartido entre maestras y niños sobre lo dicho en un texto narrativo.

\section{Metodología}

\section{Tipo de estudio}

Se trata de un estudio de tipo descriptivo-exploratorio.

\section{Participantes}

Participaron en el estudio cuatro grupos de niños de preescolar, del grado Transición, y sus respectivas maestras. La población participante procede de cuatro instituciones educativas (dos de Ibagué y dos de Cali), elegidas por muestreo intencional, ya que las maestras de Cali han sido formadas en la práctica textual, mientras que las de los grupos de Ibagué no. Para realizar las observaciones, se solicitó a las maestras que trabajarán únicamente con un grupo de 9 a 12 niños, escogidos aleatoriamente de sus grupos de clase; el muestreo utilizado fue el aleatorio simple. Con el tamaño del grupo se esperaba que las interacciones se centraran en la comprensión y que no se presentaran diferencias en cada grupo en razón del número de integrantes. Un total de 44 niños participó en el estudio.

\section{Instrumentos}

Para la recolección de la información, se utilizó la observación natural y su respectiva grabación en video. Para contar con elementos de juicio en el momento de analizar los datos, se hizo un análisis textual del cuento y de las exigencias cognitivas que comporta su comprensión en relación con la elaboración de inferencias. El texto narrativo seleccionado para la tarea de comprensión textual fue Niña Bonita de Ana María Machado.

\section{Procedimiento}

Para afinar el diseño y la propuesta de categorías de análisis, se hizo un estudio piloto y una réplica con algunas modificaciones de la investigación de
Saracho (2002), que permiten delimitar los aspectos metodológicos y así garantizar decisiones adecuadas en el estudio del problema de investigación propuesto.

Posteriormente, se realizaron los contactos con cada colegio. Se les propuso a las maestras participantes que leyeran el cuento y lo trabajarán durante tres sesiones: la primera dedicada a la lectura y las otras dos a la discusión de la historia con los niños, para buscar su comprensión. A las maestras no se les dio ninguna instrucción adicional. Las grabaciones de las dos sesiones dedicadas a la comprensión fueron transcritas; en el registro se consignaron aquellas interacciones realizadas por la maestra y los niños, cuya finalidad fuera la comprensión del texto narrativo. Cada sesión tuvo una duración promedio de 35 minutos.

Las producciones de los participantes se analizaron de acuerdo con la metodología del análisis del discurso. Los discursos se segmentaron en secuencias, teniendo en cuenta las siguientes condiciones: 1) las interacciones cuya finalidad es la comprensión del texto narrativo; 2) secuencias con un inicio y un cierre -cuando uno de los participantes comienza o termina la interacción para lograr la comprensión del texto en un mismo eje temático- y 3) la codificación por jueces. La codificación de las observaciones estuvo a cargo de cuatro personas debidamente entrenadas, tanto en observación como en la teoría que sustenta la investigación y la metodología de análisis de discurso. La confiabilidad interna interjueces fue de 0.78 .

De manera congruente con el marco conceptual del estudio, se utilizó un análisis de redes sociales. Para llevar a cabo este tipo de procesamiento de los datos, se hizo uso de un software para análisis de redes sociales, el UCINET 6 for Windows, creado por Borgatti, Everett y Freeman (2002).

Para responder a la pregunta que guía la investigación, se analizaron dos dimensiones. La Dimensión Uno corresponde a aquellas interacciones triádicas (docente-niños-cuento) o (niño-niño-cuento) que buscan la comprensión textual, y la Dimensión Dos corresponde a la comprensión inferencial del texto narrativo en los niños. 
Dimensión Uno: Interacciones tríadicas que buscan la comprensión textual

- Interacción Tipo 1 (IT1). El texto tiene sentido por sí mismo. Es aquella comprensión que se logra una vez el lector se ha apropiado del código, pues la información explícita está en el texto mismo. Los niños y la maestra recuperan información explícita sobre el narrador, tiempo de la historia, espacios en la historia, personajes, características de los personajes, relación entre personajes, acciones que realizan los personajes y conflicto(s) o problema(s) planteado(s) en la historia.

- Interacción Tipo 2 (IT2). El (los) sentido(s) del texto se da(n) a partir de las transacciones entre lector y texto. La comprensión del texto narrativo es entendida como aquella que se logra a partir de las transacciones y negociaciones realizadas entre el lector y el texto. Plantea interrogantes y observaciones sobre el texto propuesto y pretende buscar el tipo de significados, intenciones y relaciones causales que el niño identifica en el texto narrativo. Se asume que la comprensión lleva a la representación mental de muchas situaciones del mundo posible descritas en el texto.

\section{Dimensión Dos: Comprensión inferencial} del texto narrativo en los niños

Las categorías para analizar la Dimensión Dos parten de la teoría construccionista (Graesser, Lang \& Roberts, 1991; Graesser \& Wiemer-Hastings, 1999; Zwaan et al., 2005). Esta dimensión presenta $10 \mathrm{ca}$ tegorías que corresponden a los tipos de inferencias; los tres indicadores de cada categoría fueron los del estudio de Perrusi y Galvao (1998): inferencias generales, inferencias precisas e inferencias incongruentes. El primero se refiere a aquellas inferencias que corresponden con el texto leído, pero no exactamente con lo que se pregunta; el segundo, a aquellas inferencias que corresponden a lo que se pregunta según el texto leído y los conocimientos previos del estudiante y el tercero se refiere a las respuestas que no corresponden con el texto leído.

\section{Resultados}

Caracterización y tipificación de

las interacciones en el aula de clase durante actividades para lograr la comprensión de un texto narrativo

En los grados de Transición de los Colegios 1 y 2 (Ibagué), la gran mayoría de las interacciones entre la maestra y los niños fueron de Tipo 1, recuperándose fundamentalmente en estas información explícita del mismo. En ambos colegios la maestra tiene la palabra la mayor parte del tiempo y las intervenciones de los niños generalmente son para dar respuestas monosilábicas; prácticamente no ocurren interacciones entre los niños, y las que se dan entre maestra y niños se caracterizan porque son de corta duración y giran en torno a preguntas del contenido literal del texto.

Con respecto al grado Transición del Colegio 3 (Cali), en la mayoría de las interacciones entre la maestra y los niños se asume una comprensión como repetición, pero también se presentan interacciones que buscan una comprensión como transacción. La maestra regula las participaciones promoviendo la intervención de la mayoría de los niños; aunque las interacciones son principalmente docente-niños en forma de pregunta-respuesta y de corta duración, se presentan algunos comentarios espontáneos e interacciones niño-niño.

Finalmente, la dinámica interactiva en el grado Transición del Colegio 4 (Cali) es diferente a la de los otros colegios; se alternan interacciones que propician una comprensión como repetición e interacciones que buscan una comprensión como transacción, con más indicadores de esta última. En relación con la interacción niño-niño, se presentan siete interacciones: tres que buscan una comprensión como repetición y cuatro, una comprensión como transacción. Las interacciones maestra-niños y niños-niños son de larga duración e involucran el diálogo, cuyo contenido gira en torno tanto a lo literal como a los significados implícitos del cuento. 


\section{Tipificación de las inferencias que elaboran los niños durante actividades para lograr la comprensión de un texto narrativo}

La Figura 1 muestra el análisis de los datos en los cuatro grados de transición de los colegios, en términos de los 10 tipos de inferencia que se pueden hacer de un texto narrativo, considerando que cada una de ellas puede ser precisa o general, dependiendo del grado de elaboración.

En la Figura 1, se evidencia que en los Colegios 1 y 2 hay una pobre presencia de inferencias en los niños, y que en ambos elaboran inferencias referenciales y de reacciones emocionales. En los Colegios 3 y 4, hay mayor elaboración inferencial en los niños, tanto por su número como por su variedad, según la tipología propuesta.

\section{Relaciones posibles entre los tipos de inferencias de los niños y los tipos de interacciones para lograr la comprensión del texto narrativo}

Para cada sesión por grado de Transición se propone una gráfica de redes sociales. En dichas gráficas, cada red representa un tipo de interacción en cada sesión (interacciones que buscan una comprensión como repetición e interacciones que buscan una comprensión como transacción). Los nodos representan a los sujetos que participaron en la sesión: la docente (Maestra) y cada uno de los estudiantes de transición (Sn); el tamaño del nodo indica el número de inferencias que realizó el sujeto (es decir entre mayor sea el tamaño del nodo mayor es el número de inferencias). Cada línea entre dos nodos (sujetos) representa la existencia de vínculo entre ellos; el grosor y el número sobre la línea representan el número de vínculos entre dos nodos (sujetos). Seguidamente, se presentan dos ejemplos de gráficas de redes sociales y el balance general por colegio de la relación entre las Dimensiones Uno y Dos. Este tipo de gráfica evidencia que el nodo que representa a la maestra siempre se ubica en el centro, lo que implica que es ella la que media entre los niños y el texto y entre niño-niño y texto.

\section{Balance del Colegio 1 (Ibagué)}

Las tres gráficas de la interacción Tipo 1 de este colegio son muy similares. La docente interactúa con todos los que asisten a la sesión, pero con mayor frecuencia lo hace con S1, S2 y S6; además, con respecto a la interacción como transacción, dos de

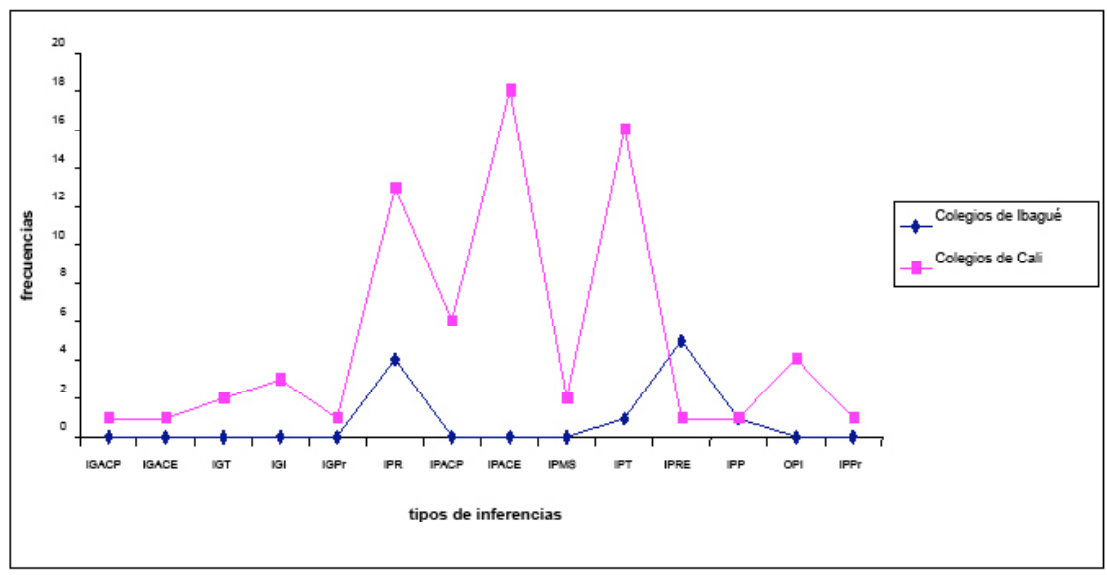

Figura 1. Tipos de Inferencias que elaboran los niños en los cuatro colegios de la muestra.

Nota. IGACP: Inferencia general antecedente causal puente; IGACE: inf. General antecedente causal elaborativa; IGT: inf. General temática; IGI: inf. General instrumental; IGPr: inf. General predictiva; IPR: inf. Precisa referencial; IPACP: inf. Precisa antecedente causal puente; IPACE: inf. Precisa antecedente causal elaborativa; IPMS: inf. Precisa meta superordinada; IPT: inf. Precisa temática; IPRE: inf. Precisa reacciones emocionales; IPP: inf. Precisa pragmática; IPI: Inf. Precisa instrumental; IPPr: inf. Precisa predictiva.

Fuente: elaboración propia. 


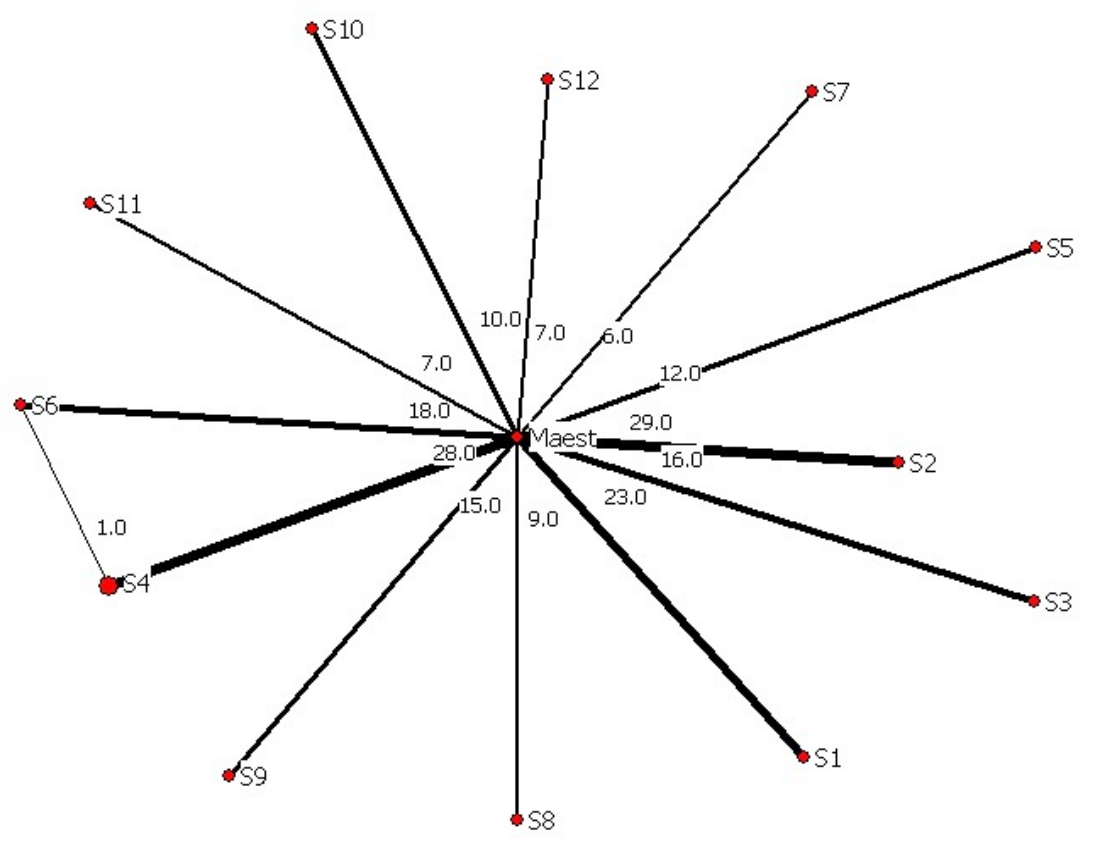

Figura 2. Relación entre interacción Tipo 1 e inferencias en la sesión 1 del Colegio 1.

Fuente: elaboración propia.

las tres sesiones son idénticas, solamente se presenta una interacción con la misma estudiante (S2), con dos inferencias hechas por la niña. Se evidencia una interacción muy limitada niño-niño. A continuación, se presenta una gráfica de redes sociales del Colegio 1 (Figura 2).

\section{Balance del Colegio 2 (Ibagué)}

Con respecto al Colegio 2 de Ibagué, se puede decir que las tres gráficas de la interacción como repetición son muy similares. La maestra interactúa con todos los asistentes a las sesiones, pero en mayor medida con dos niños. En este tipo de interacción solamente se presenta una inferencia. Con respecto a las interacciones como transacción, la dinámica es diferente en las tres sesiones: en la primera, la docente interactúa con cuatro de los estudiantes; en otra, solo con una niña y en la tercera sesión no hay interacción como transacción. De las nueve interacciones Tipo 2 que se presentan, se elaboran 6 inferencias precisas.
Balance del Colegio 3 (Cali)

Con respecto al Colegio 3 de Cali, las gráficas de la interacción como repetición en las tres sesiones son muy similares. La docente interactúa con todos los estudiantes asistentes a la sesión; solo se elaboran inferencias en la sesión 2; los niños con los que más interactúa la maestra son S1, S2, S7 y S9; únicamente se presentan tres interacciones niño-niño. Con relación a la interacción como transacción, las gráficas varían: en la primera sesión interactúan pocos niños con la docente y ya en la sesión 2 aumentan las interacciones; finalmente, en la sesión 3, todos, menos dos niños, tienen interacción Tipo 2 con la maestra; de esta interacción resulta en total 12 inferencias; los sujetos con los que más interactúa son $\mathrm{S} 7$ y S10, siendo S7 uno de los que más elabora inferencias.

\section{Balance del Colegio 4 (Cali)}

En el Colegio 4 es donde ocurren más interacciones como transacción, entre niño-niño, y elaboraciones 


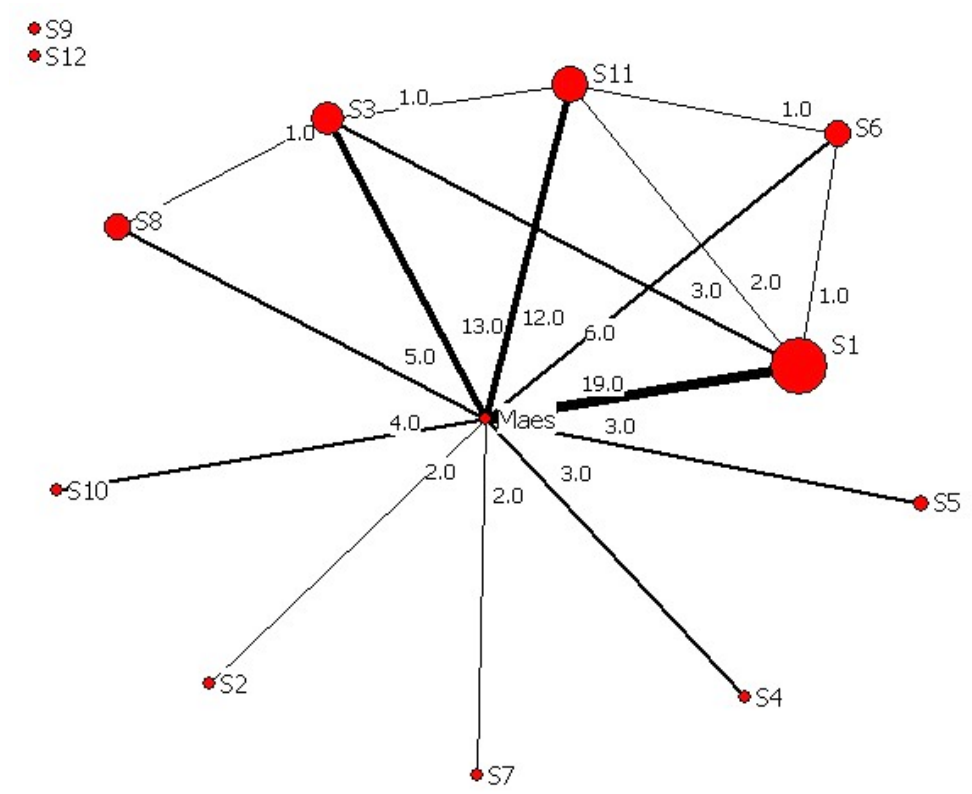

Figura 3. Relación entre interacción Tipo 2 e inferencias en la sesión 2 del Colegio 4.

Fuente: elaboración propia.

inferenciales de naturaleza más variada. La maestra interactúa con todos los niños asistentes a clase, aunque lo hace en mayor frecuencia con S1, S3, S5 y S11; además, en las tres sesiones se da la interacción niño-niño. Estas interacciones como transacción posibilitan en total 54 inferencias, 6 generales y 48 precisas; los niños que más inferencias formulan son S1, S3 y S11, y estos niños también son los que más interactúan con la maestra; la mayoría de los niños elaboran por lo menos una inferencia.

La gráfica de la Figura 3 permite observar que en las interacciones se dan 35 inferencias, 9 de estas en interacciones niño-niño; el S1 elabora 12 inferencias precisas; el S3 elabora 10 IP; además, S11 elabora 6 IP; también S6, S8 y S5 elaboran inferencias.

Con respecto a las interacciones como repetición en este grado de preescolar, se encuentra que son muy similares en las tres sesiones: la maestra interactúa con todos los niños y hay pocas interacciones niño-niño; los niños que más participan en este tipo de interacción son: S1 y S3; no hay elaboración de inferencias en estas interacciones.

\section{Discusión}

Ya se ha dicho que el objetivo principal del estudio es establecer las posibles relaciones entre las inferencias que elaboran los niños sobre un texto narrativo y las características de las interacciones que se dan en el espacio de la clase. A partir del análisis de redes sociales, se encuentra una relación entre pobre elaboración inferencial-bajo número de inferencias formuladas, tipo de inferencias: las menos complejas y poca variedad inferencial- y el predominio de las interacciones que buscan una comprensión como repetición del contenido explícito del texto, que asumen que el sentido del texto está solo en él. Los resultados muestran que existe relación entre mayor número de interacciones como transacción, que buscan una comprensión como búsqueda del (los) sentido(s) del texto, y la frecuencia de las inferencias elaboradas por los niños, su mayor riqueza, complejidad y variedad.

La comprensión de textos narrativos requiere que se dé una serie de condiciones -ya señaladas-, en 
la interacción entre el adulto y el niño. En el grado de Transición, el rol de la maestra es central en el acercamiento a los mundos posibles propuestos en los textos narrativos. Dadas las características de los niños de cinco años, es el adulto el que da la pauta y posibilita ciertos tipos de interacciones (Berk, 1999). Los hallazgos del estudio reafirman lo encontrado en investigaciones anteriores (González, 2005; Moschovaki \& Meadows, 2005), en las que se señala que las participaciones con una demanda cognitiva alta por parte de la maestra, promueven iguales participaciones en los niños.

Las maestras con más indicadores de interacciones como transacción y con mayor elaboración inferencial por parte de los niños, son las de los Colegios 3 y 4 , que se han formado desde la perspectiva conceptual de la práctica textual. Dado que el estudio realizado no busca, por el diseño y tamaño de la muestra, hacer generalizaciones, no se puede afirmar que las diferencias de elaboración inferencial encontradas en los niños se deban únicamente a este aspecto, solo se puede postular que parece haber una relación entre ambos factores y que se requiere de otros estudios que indaguen la variable formación de la maestra y su incidencia en los funcionamientos cognitivos de alto orden que presenten los niños. La maestra del Colegio 4 refleja una postura en la que asume que el salón de clases es un espacio de negociación de significados entre la maestra y sus estudiantes y entre los estudiantes mismos, en torno a un objeto de conocimiento: el texto narrativo (Bruner, 1997; Correa, 2006).

Adicionalmente, los resultados encontrados en la investigación apoyan los datos de otros estudios (Duque \& Vera, 2010; Espéret \& Fayol, 1997; González, 2005; Jiménez \& Marmolejo, 2007, Moschovaki \& Meadows, 2005; Ordóñez \& Bustamante, 2000; Perrusi \& Galvao, 1998) respecto a que los niños de preescolar de cinco años están en capacidad de formular inferencias de diferente complejidad y tipología.

Las inferencias que se encuentran en los niños de todos los colegios son las referenciales, y en tres de los cuatro colegios las de reacciones emocionales de los personajes; mientras que en los niños del Colegio 4 se presentan todas las categorías inferenciales, menos la de consecuente causal. En los niños de los Colegios 1 y 2, hay una pobre elaboración de inferencias y en ambos se formulan solo algunas inferencias referenciales y de reacciones emocionales. Este tipo de inferencias son más fáciles de hacer en esta edad (Duke, Pressley \& Hilden, 2004; Jiménez \& Marmolejo, 2007; Ordóñez \& Bustamante, 2000). También algunas características del texto favorecen que los niños elaboren estas inferencias (Espéret \& Fayol, 1997; GutiérrezCalvo, 1999; Zwaan \& Madden, 2004).

En los Colegios 3 y 4, los niños elaboran inferencias causales. Estas son las inferencias centrales para la comprensión de textos, porque permiten establecer coherencia tanto local como global (Duke et al, 2004; Espéret \& Fayol, 1997; Goldman et al., 1999; Graesser, Wiemer-Hastings, 1999; Graesser \& Zwaan, 1995, Gutiérrez-Calvo, 1999; León, 2001; Magliano, 1999; Perrusi \& Galvao, 1998). Los niños del Colegio 4 son los que presentan más elaboraciones inferenciales; las de mayor frecuencia son las causales elaborativas y temáticas; estas últimas también son centrales para lograr la comprensión global del texto (Duke et al., 2004).

Si se tiene en cuenta que la elaboración de inferencias en relación con los textos narrativos ocurre ya en los niños menores de cinco años, es primordial que las maestras y maestros creen situaciones y formas de aproximarse a esta capacidad, de tal forma que la demanden y propicien. Una de estas es la valoración de actuación, en la que se realizan discusiones en grupo en torno al texto, exigiéndoles a los estudiantes que generen productos a partir del texto y que demuestren sus conocimientos (Elliot, 1998 citado por Duke et al., 2004). Esta forma de aproximarse al texto se refleja especialmente en la maestra del Colegio 4.

Duke et al. (2004) y Stone, Silliman, Ehren y Apel (2004) consideran que el desafío más grande está en que los docentes entiendan que el proceso de comprensión de lectura es complejo. En primer lugar, porque convergen en la comprensión diferentes funcionamientos cognitivos que requieren condiciones propicias para que se desplieguen; en segundo término, es un proceso que se presenta de manera diferente en cada individuo, porque depende de las características de los niños y de los factores instruc- 
cionales que lo median. Por lo mismo, es indispensable que las y los maestros tengan la posibilidad de formarse en prácticas que les permitan aproximar a los niños a la lectura de manera adecuada.

Formulaciones hechas desde perspectivas socioculturales (Mercer, 2001; Vigotsky, 1987; Villegas, 2006) asumen que el desarrollo es un proceso social y, por ende, el conocimiento se da a partir de la interacción y posterior internalización del conocimiento hecha por el niño. Por su parte, Valsiner (2004) ha llamado a esta co-construcción "proceso bidireccional de transmisión cultural"; además, Bruner (1997) indica que la comprensión es un proceso conjunto entre dos o más miembros, por lo tanto, en esta co-construcción se da una co-regulación que guía patrones de acción y comunicación hacía la autoorganización (Van Geert, 1994). Por tales razones, esta investigación tendría implicaciones para otros contextos educativos, diferentes a los propuestos en el estudio, porque les permite a quienes estén interesados en mejorar la comprensión textual en lectores iniciales, tener en cuenta el papel decisivo de las características de la interacción en contextos de aula.

Aunque no se puede afirmar que sea únicamente responsabilidad de la maestra o el maestro que el niño elabore o no inferencias a partir del texto, su papel es central. Se requiere de maestros que acepten el reto de modificar prácticas en el aula, para promover la lectura comprensiva en sus diferentes niveles. Lo anterior hace necesario seguir estudiando las interacciones que se presentan alrededor del texto narrativo en el salón de clase, para relacionar esta dimensión con la comprensión textual. Sería importante realizar ulteriores estudios que permitan establecer relaciones y hacer predicciones respecto a cómo ciertas prácticas pedagógicas posibilitan funcionamientos cognitivos de alto nivel en los estudiantes en relación con la comprensión textual.

\section{Referencias}

Belinchón, M., Rivière, A. \& Igoa, J. M. (2000). La comprensión del discurso. En M. Belinchón, A. Rivière \& J. M. Igoa (Eds.), Psicología del lenguaje. Investigación y teoría (pp. 181-235). Madrid: Trotta.
Berk, L. (1999). Desarrollo del niño y del adolescente (4. ed.). Madrid: Prentice Hall Iberia.

Bobbitt, N. S. (2001).Constructing literacy in the kindergarten: Task structure, collaboration and motivation. Cognition and Instruction, 19(1), 95-142.

Borgatti, S. P., Everett, M. G. \& Freeman, L. C. (2002). Ucinet for Windows (Software for Social Network Analysis). Harvard, MA: Analytic Technologies.

Bravo, L., Villalón, M. \& Orellana, E. (2004). Los procesos cognitivos y el aprendizaje de la lectura inicial: diferencias cognitivas entre buenos lectores y lectores deficientes. Estudios Pedagógicos, 30, 7-19.

Bravo, L., Villalón, M. \& Orellana, E. (2006). Predictibilidad del rendimiento en la lectura: una investigación de seguimiento entre primer y tercer año. Revista Latinoamericana de Psicología, 38(1), 9-20.

Bruner, J. (1995). Actos de significado. Más allá de la revolución cognitiva. Madrid: Alianza.

Bruner, J. (1997). La educación, puerta de la cultura. Madrid: Visor.

Cerrillo, P., Larrañaga, E. \& Yubero, S. (2002). Evolución psicológica y maduración lectora. En Libros, lectores y mediadores (pp. 13-27). Cuenca: Ediciones de la Universidad de Castilla-La Mancha.

Correa, M. (2003). Yo me sé el cuento de... En B. Orozco (Comp.), El niño: científico, lector y escritor, matemático (pp. 99-135). Cali, Col: Universidad del Valle.

Correa, M. (2006). Contexto, interacción y conocimiento en el aula. Pensamiento Psicológico, 2(7), 133-148.

Correa, M., Orozco, B. \& Conde, E. (1999). Guía conceptual para trabajar el video. La anticipación de la estructura narrativa: una estrategía para la enseñanza de la lectura y la escritura. Cali, Col: Universidad del Valle/Colciencias.

Duke, N., Pressley, M. \& Hilden, K. (2004). Difficulties with reading comprehension. En C. Stone, E. Silliman, B. Ehren \& K. Apel (Eds.), Handbook of language and literacy: Development and disorders (pp. 501-520). New York: Guilford.

Duque, C. (2006). Conciliando el aprendizaje formal e informal de la lectura emergente en contextos escolares. Revista Colombiana de Psicología, 15, 125-129.

Duque, C. \& Vera, A. (2010). Exploración de la comprensión inferencial de textos narrativos en niños 
de preescolar. Revista Colombiana de Psicología, 19, 21- 35.

Duque, C., Vera, A. \& Hernández, A. (2010). Comprensión inferencial de textos narrativos en primeros lectores: una revisión de la literatura. Ocnos: Revista de Estudios sobre Lectura, 6, 35-44.

Eco, U. (1996). Seis paseos por los bosques narrativos. Barcelona: Lumen.

Espéret, E. \& Fayol, M. (1997). Producción y comprensión del lenguaje escrito. En G. Vergnaud (Coord.), Aprendizaje y didácticas: iqué hay de nuevo? (pp. 41-60). Buenos Aires: Edicial.

Flórez, R., Torrado, M. C., Arévalo, I., Quintero, C. \& Rodríguez, O. (2008). Promoción del alfabetismo emergente y prevención de las dificultades en la lectura: una experiencia pedagógica en el aula. Santa Marta, Col: Universidad del Magdalena. Recuperado el 15 de junio de 2009, de http://www.ascofapsi. org.co/encuentro_investigadores/grupos_investigacion_psicologia.pdf

Goldman, S., Varma, K., Sharp. D. \& Cognition and Technology Group at Vanderbilt. (1999). En S. R. Goldman, A. C. Graesser \& P. van den Broek (Eds.), Narrative comprehension, causality, and coherence: Essays in honor of Tom Trabasso (pp. 135 159). Mahwah, NJ: Erlbaum.

González, G. J. (2005). Elaboración conjunta de inferencias a partir de cuentos infantiles. Psicología Educativa, 11(2), 113-133.

Goodman, Y. M. (1991). Descubriendo la invención de la lengua escrita en los niños. Buenos Aires: Aique.

Graesser, A. C., Lang, K. L. \& Roberts, R. M. (1991). Questions answering in the context of stories. Journal of Experimental Psychology: General, 120(3), 254-277.

Graesser, A. C. \& Wiemer-Hastings, K. (1999). Situation models and concepts in story comprehension. En S. R. Goldman, A. C. Graesser \& P. van den Broek (Eds.), Narrative comprehension, causality, and coherence: Essays in honor of Tom Trabasso (pp. 77-92). Mahwah, NJ: Erlbaum.

Graesser, A. C. \& Zwaan, R. (1995). Inference generation and the construction of situation models. En C. I. Weaver, S. Mannes \& C. Fletcher (Eds.), Discourse comprehension. Essays in honor of Walter Kintsch (pp. 117-139). Hillsdale, NJ: Erlbaum.
Gutiérrez-Calvo, M. (1999). Inferencias en la comprensión del lenguaje. En M. Y. De Vega \& F. Cuetos (Eds.), Psicolingüística del español (pp. 231-270). Madrid: Trotta.

Jiménez, A. \& Marmolejo, F. (2007). Inferencias, modelos de situación y emociones en textos narrativos. El caso de los niños de preescolar. Revista Intercontinental de Psicología y Educación, 8(2), 93-138.

Jouini, K. \& Saud, K. (2005). Estrategias inferenciales en la comprensión lectora. Glosas Didácticas, 13, 96-115. Disponible en http://www.um.es/glosasdidacticas/GD13/GD13INDEDIT.pdf

Jurado, V. F., Bustamante, Z. G. \& Pérez, A. M. (1998). Juguemos a interpretar: evaluación de competencias en lectura y escritura. Bogotá: Plaza \& Janés.

León, J. (2001). Las inferencias en la comprensión e interpretación del discurso. Un análisis para su estudio e investigación. Revista Electrónica de Investigación Educativa, 34, 113-125.

Magliano, J. (1999). Revealing inference processes during text comprehension. En S. R. Goldman, A. C. Graesser \& P. van den Broek (Eds.), Narrative comprehension, causality, and coherence: Essays in honor of Tom Trabasso (pp. 55-75). Mahwah, NJ: Erlbaum.

Mercer, N. (2001). Palabras y mentes. Barcelona: Paidós. Moschovaki, E. \& Meadows, S. (2005, primavera). La participación espontánea de los niños pequeños durante la lectura de libros en el aula. Diferencias según el tipo de libro. Early Childhood Research \& Practice, 7(1). Recuperado el 15 de enero de 2009, de http://ecrp.uiuc.edu/v7n2/moschovaki-sp.html

Ordóñez, O. A. \& Bustamante, L. G. (2000). Habilidades para la comprensión y el razonamiento científico en el niño. Una revisión bibliográfica. En R. Puche-Navarro (Ed.), Formación de herramientas científicas en el niño pequeño (pp. 141-182). Bogotá: Arango Editores.

Orozco, B. C. (2003). iLee el niño preescolar? En B. C. Orozco (Comp.), El niño: científico, lector y escritor, matemático (pp. 75-97). Cali, Col: Artes Gráficas del Valle.

Orozco, B., Puche, R., Millán, R. \& Rojas, T. (2004). Inferencia, comprensión de textos narrativos y formación de herramientas científicas en niños invidentes 
(Informe final de proyecto). Cali, Col: Universidad del Valle.

Perrusi, B. \& Galvao, S. (1998). Aspectos generales y especificos en la producción de textos. Recife, $\mathrm{Br}$ : Universidad Federal de Pernambuco.

Rincón, G., Narváez, E. \& Roldán, C. (2005). Enseñar a comprender textos en la universidad: análisis de dos casos. Cali, Col: Unidad de Artes Gráficas del Valle.

Saracho, O. N. (2002). Teachers' roles in promoting literacy in the context of play. Early Child Development and Care, 172(1), 23-34.

Scardamalia, M. \& Bereiter, C. (1992). Dos modelos explicativos de los modelos de comprensión escrita. Infancia y Aprendizaje, 58, 43-64.

Stahl, S. A. \& Yaden, D. B., Jr. (2004). The development of literacy in preschool and primary grades: Work by the Center for the Improvement of Early Reading Achievement. Elementary School Journal, 105(2), 141-165.

Stone, C., Silliman, E., Ehren, B. \& Apel, K. (Eds.). (2004). Handbook of language and literacy: Development and disorders. New York: Guilford.
Teberosky, A. \& Tolchinsky, L. (1998). Más allá de la alfabetización. Buenos Aires: Santillana.

Van Dijk, T. A. \& Kintsch, W. (1983). Strategies of discourse comprehension. New York: Academic Press.

Valsiner, J. (2004). El desarrollo de las teorías del desarrollo: "la hollywoodización" de la ciencia y su impacto. Infancia y Aprendizaje, 27, 147-154.

Vigotsky, L. S. (1987). Pensamiento y lenguaje. Buenos Aires: Editorial La Pléyade.

Villegas, R. O. (1996). Seminario-Taller: proyecto pedagógico- lengua materna en los grados obligatorios: 1 , 2 y 3. Manuscrito no publicado, Universidad del Valle, Cali, Colombia.

Van Geert, P. (1994). The dynamics of mutual interaction. En Dynamic systems of development: Change between complexity and chaos (pp. 242-277). New York: Harvester Wheatsheaf.

Zwaan, R. A. \& Madden, C. (2004). Updating situation models. Journal of Experimental Psychology: Learning, Memory \& Cognition, 30(1), 283-288.

Zwaan, R. A., van den Broek, P. \& Sundermeier, B. (2005). Causal coherence and the accessibility of object locations in narrative comprehension. Memory \& Cognition, 33(3), 462-470. 